\title{
Droplet Spreading Characteristics Observed During 3D Printing of Aligned Fiber-reinforced Soft Composites
}

\author{
Kyle Picha \\ Graduate Research Assistant \\ pichak@rpi.edu
}

\author{
Clayson Spackman \\ Graduate Research Assistant \\ spackc@rpi.edu
}

\author{
Johnson Samuel ${ }^{1}$ \\ Assistant Professor \\ samuej2@rpi.edu
}

Department of Mechanical Aerospace and Nuclear Engineering, Rensselaer Polytechnic Institute, $1108^{\text {th }}$ Street, Troy, NY
12180, USA

\begin{abstract}
The 3D printing of fiber-reinforced soft composites (FrSCs) is a hybrid process that combines conventional inkjet-based 3D printing with the directed deposition of electrospun polymer fiber mats. This paper investigates the spreading characteristics of droplets when deposited on fibrous substrates, under conditions relevant to 3D printing of aligned FrSCs. Both single and multidroplet impingement studies are conducted on substrates with varying fiber number densities. High-speed imaging is used to study the characteristic time-scales and the spreading behavior of the droplets. The single droplet impingement studies on stationary substrates reveal that the presence of fibers promotes droplet spreading along the length the fibers. Occasional surface energy variations in the fiber mats in the form of voids and fiber bundles are also seen to affect the droplet shape and the characteristic spreading times. In the case of a moving substrate, the droplets are seen to spread the most during in-line printing, i.e., when the direction of the printing velocity coincides with the direction of fiber alignment. They spread the least during orthogonal printing, i.e., when the direction of the printing velocity is perpendicular to the direction of fiber alignment. The printing of straight lines shows an interesting edge retraction phenomenon that gets accentuated the most in the case of in-line printing. The findings of the high-speed imaging studies have been confirmed by 3D printing comparable artifacts using UV curable inks. These studies indicate that for a given fiber mat and UV curable ink combination, the choice of the in-line or orthogonal printing strategy has implications for the overall printing time, fiber content, edge resolution and surface quality of the 3D printed FrSC part.
\end{abstract}

Keywords: Fiber-reinforced soft composites; Inkjet printing; Additive manufacturing; 3D printing; Droplet spreading

\section{Introduction}

Fiber-reinforced soft composites (FrSCs) are a new class of composite materials made up of hierarchical, polymer fiber networks embedded within another soft polymer matrix [1-2]. The applications of FrSCs include areas such as 4D printing, bio-mimetic materials, artificial muscles, sensors and actuators [3-5]. Current 3D printing processes such as fused deposition modeling [6], stereolithography [7], and inkjet-based deposition of UV curable polymers [8] are not capable of printing FrSCs. Recently, Spackman et al. [9] developed a novel hybrid 3D printing process to manufacture FrSCs. This process combines the conventional inkjet-based 3D printing technique with the directed deposition of micro/sub-micron fibrous structures manufactured using electrospinning. While this process has been proven to 3D print FrSCs, the interactions between the droplets (ejected by the inkjet nozzle) and the fiber mats are not well understood. This understanding is critical to the development of process control strategies for 3D printing FrSCs.

The study of the interaction between droplets and fibrous substrates dates back to the classic Cassie-Baxter and Wenzel models that link contact angles and wetting regimes to surface roughness parameters [10,11]. More recent investigations have included the study of droplets spreading on foams [12], powder beds [13], textiles [14], and on microfibers [15-17]. There have also been studies concerning micro-scale droplets deposited on wrinkled or grooved substrates [18]. However, the substrate scenarios in these studies are not comparable to the multi-material, fibrous surfaces encountered during the 3D printing of FrSCs [9].

The objective of this paper is to study the spreading characteristics of droplets when deposited onto fibrous substrates, under conditions relevant to the domain of 3D printing FrSCs. Both single and multi-droplet impingement studies are conducted on different fibrous substrates using an inkjet-based droplet deposition system. High-speed imaging is used to study the characteristic time-scales and the spreading behavior of the droplets. The findings enable an effective understanding of the process planning issues relevant to the 3D printing of FrSCs.

The remainder of this paper is organized as follows. Section 2 provides an overview of the relevant background literature concerning FrSCs and droplet spreading regimes. Section 3 describes the high-speed imaging setup details. Sections 4 and 5 discuss the findings from the single droplet impingement studies on stationary and moving substrates. Section 6 discusses the results seen

\footnotetext{
${ }^{1}$ Corresponding author email: samuej2@rpi.edu
} 
while printing lines using overlapping droplets. Sections 7 and 8 translate these findings to the printing performance of the FrSC 3D printer. Finally, Section 9 presents the specific conclusions that can be drawn from this study.

\section{Background Literature Review}

This section will provide a brief overview of the relevant background literature concerning the FrSC 3D printing process and the droplet spreading behavior relevant to inkjet-based printing systems.

\subsection{FrSC $3 D$ printing process}

The 3D printing of FrSCs first includes the manufacture of the fiber mats using either a near-field or far-field electrospinning-based direct-write process [9, 19]. These fiber mats are then transferred to a five-axis inkjet-based 3D printing platform. A layer-by-layer manufacturing technique is then used to 3D print the FrSC parts (Fig. 1a). This process consists of printing and curing a layer of the UV curable polymer, followed by a stamping procedure that transfers the fibrous mat onto the printed polymer layer. Once the fibers are transferred, this sequence of operations is repeated until the whole part is manufactured in the form of a laminated composite structure.

Figure $1 \mathrm{~b}$ depicts the cross-section of a 3D printed FrSC part showing the alternate layers of UV curable ink and fiber mats deposited in between each layer. Unlike the conventional inkjetbased 3D printing of UV curable polymers where the droplets are deposited only on the previously printed layer of polymer, in the case of FrSCs these droplets are deposited on fibrous mats stamped on the previously printed layer. This heterogeneous surface is expected to influence the spreading characteristics of the droplet. A critical understanding of these spreading characteristics will enable the development of novel process control strategies that will ensure the dimensional accuracy of the 3D printed FrSC part while also helping to lower printing times.

\subsection{Droplet spreading behaviors}

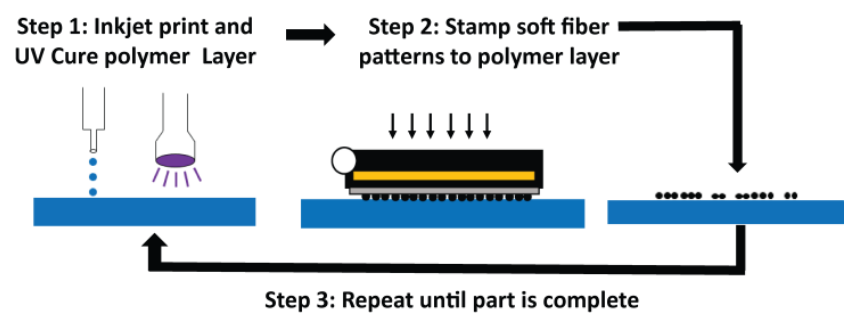

(a)

(b)

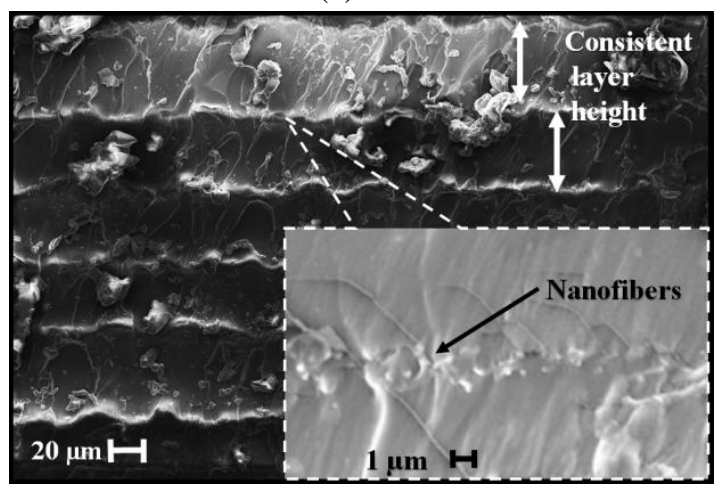

Fig. 1: Layer-by-layer manufacturing process used to 3D print FrSCs (a) Process outline (b) Cross-section of printed part [9]

This section summarizes the seminal work of Schiaffino and Sonin [20] and Rioboo et al. [21], both of which form the foundation of inkjet-based droplet spreading studies reported in literature [22,23]. The work of Schiaffino and Sonin [20] identified four regions of droplet spreading dynamics that depend on the Weber number and Ohnesorge number of the fluid. The Weber number (We) describes the driving force of droplet spreading, while the Ohnesorge number $(O h)$ describes the resistance to spreading [20], and are given by the expressions $\rho V^{2} D_{0} / \sigma$ and $\mu / \sqrt{\rho \sigma D_{0}}$, respectively, where $\rho$ is the fluid density, $V$ is the droplet velocity, $D_{0}$ is the original droplet diameter, $\sigma$ is the fluid surface tension, and $\mu$ is the fluid viscosity. Figure 2a shows the location of these four regions, with characteristic transitions at We and $\mathrm{Oh}$ number values of 1 . Most inkjet-based droplet-on-demand printing systems fall in Region 1 (We $>1$ and $\mathrm{Oh}<1$ ), which is confirmed by the * markers on Fig. 2a, denoting the four inks used in this paper. In Region 1, the spreading is primarily driven by the inertial energy of impact [24].

Rioboo et al. [21] studied the time-dependent spreading of droplets and identified four characteristic phases. Figure $2 \mathrm{~b}$ shows a representative plot of the dimensionless spreading factor, $\beta$ (defined as $\mathrm{D} / \mathrm{D}_{0}$ where $\mathrm{D}$ is the spread diameter and $\mathrm{D}_{0}$ is the diameter of the undeformed droplet measured before impact), as a function of the dimensionless time, $t *$ (defined as $t V / D_{0}$, where $t$ is the time of spread and $V$ is the velocity of the droplet before impact). As seen in Fig. 2b, the five phases of droplet spreading include a kinematic phase followed by a spreading phase, a relaxation phase, a wetting phase and finally an equilibrium phase. The first two phases occur in the time domain $t^{*}<1$ with the relaxation phase being approximately in the time domain $1<t^{*}<10$. Since the time difference between the jetting of the droplet and the UV curing step is of the order of milliseconds, the $t^{*}$ relevant to the 3D printing of FrSCs is greater than 10. This makes the wetting phase to be the most critical in impacting the printing resolution, since the UV curing step could be turned on at any point during the spreading of the droplet in this phase (Fig. 2b). At the end of this wetting phase, the droplet reaches equilibrium, i.e., the velocity of the contact line between the droplet and the surface becomes zero. These findings of Rioboo et al. [21] and Schiaffino and Sonnin [20] have been used in several recent studies [25-29] investigating the droplet spreading phenomenon on a variety of surfaces. 

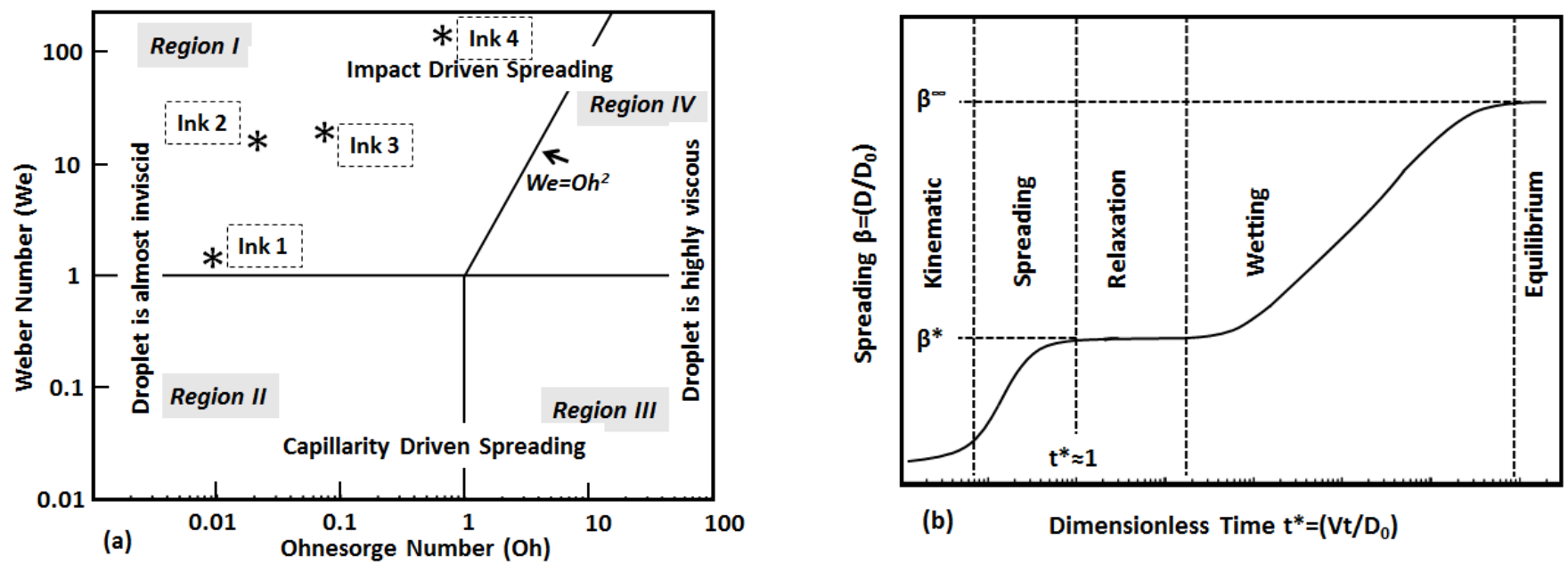

Fig. 2: Droplet spreading characteristics (a) Four regions of spreading dynamics [20] (b) Phases of droplet spreading [21]

\section{High-speed Imaging Study}

Figure 3a shows the schematic of the experimental setup used to perform the high-speed imaging studies on the droplets. The basic elements of the setup include a MicroFab ${ }^{\mathrm{TM}}$ inkjet nozzle $(120 \mu \mathrm{m}$ diameter) used for the 3D printing operation, a high-intensity Prior ${ }^{\mathrm{TM}}$ Lumen 200 light source, a glass slide substrate holder and a Phantom ${ }^{\mathrm{TM}} 7.3$ high-speed camera attached to a $20 \mathrm{X}$ Mitutoyo lens. The pneumatic controller and vacuum pump that allow for variable backpressure of the ink in the nozzle are not shown in Fig. 3a. The nozzle is operated using the MicroFab ${ }^{\mathrm{TM}}$ JetDrive system that uses specific voltage waveform patterns, in conjunction with the back pressure, to print the UV curable inks. The waveform parameters for jetting are unique to each ink, with more viscous inks requiring higher voltages to print. The nozzle can deposit the polymers in two modes, viz. the droplet-on-demand (DoD) mode and the burst mode. The DoD mode is used to print single droplets whereas the burst mode is used to print a series of continuous droplets to generate a printed line. The top view (Fig. $3 \mathrm{~b}$ ) and the front view (Fig. 3c) of the droplet is captured using a camera line-of-sight inclined at $75^{\circ}$ and $5^{\circ}$, respectively, to the substrate surface.

\subsection{Ink selection}

The use of UV curable inks for the high-speed imaging study is impractical because the wavelengths of the light emitted by the light source are capable of curing the ink in the inkjet nozzle. Therefore, a substitute ink must be used for the high-speed imaging study. This substitute ink must have viscosity and surface tension values comparable to the UV curable polymers, but it should not react to the high-intensity light used for imaging purposes. A mixture of deionized (DI) water and glycerol is one such combination that has been a proven substitute for high-speed imaging applications involving UV curable inks [30].

Four different DI water and glycerol combinations listed in Table 1 were used in this high-speed imaging study. The four inks have dynamic viscosities of $0.0009,0.0016,0.0069$, and $0.0669 \mathrm{PaS}$, and these will be referred to as Inks 1, 2, 3, and 4, respectively, for the remainder of this paper. Ink 1 is DI water and it is well-studied in literature [10,11,14-18,21,22]. Ink 2 is similar to the widely-used photoresist SU-8 2000 (MicroChem). Ink 3 is analogous to Sartomer SR531, which was used to create FrSCs by Spackman et. al [9]. Ink 4 is similar in viscosity to Stratasys TangoPlus FLX930, the ink most commonly used to 3D print FrSCs. Table 1 also lists the composition of these inks (i.e., vol.\% of glycerol and vol.\% of DI water), their surface tension
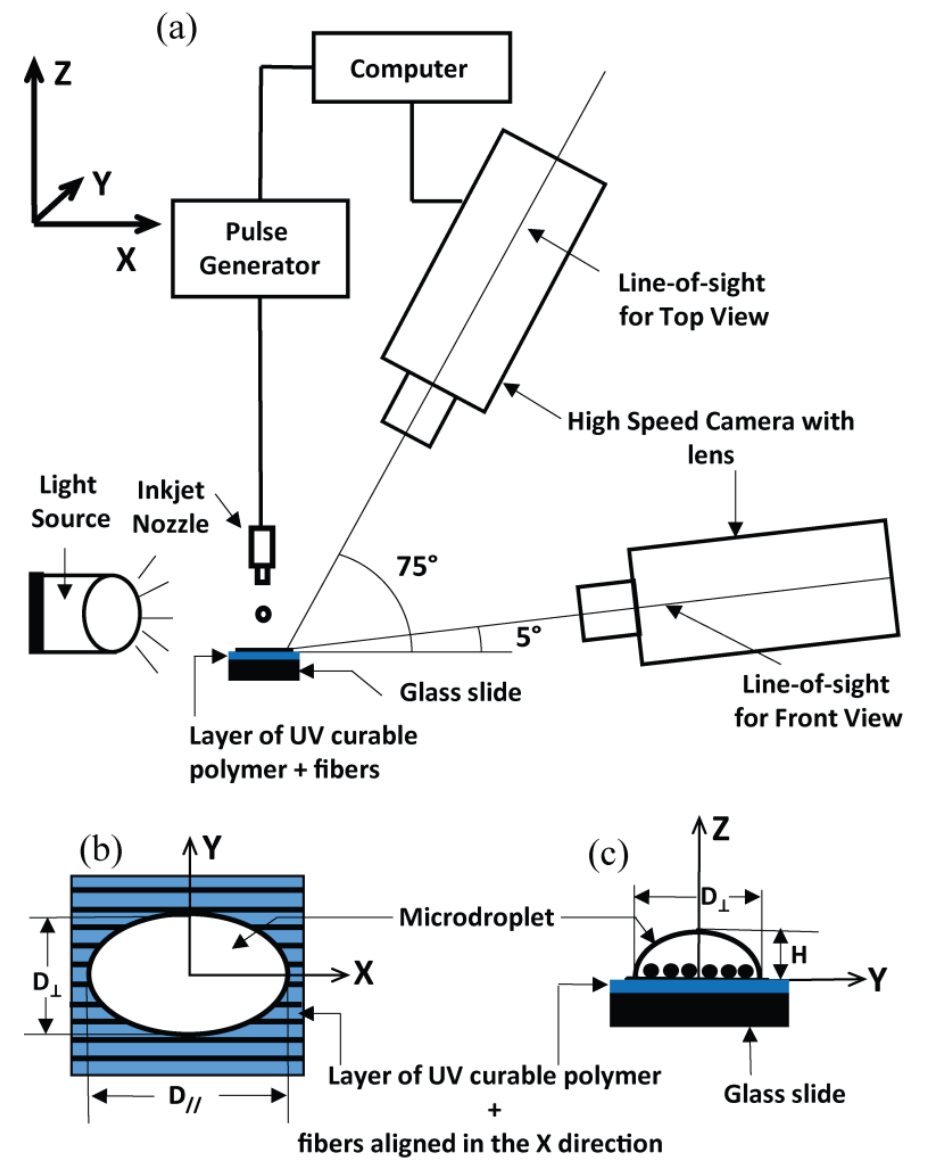

Fig. 3: Schematic of high-speed imaging setup (a) overall setup (b) top-view of the droplet (c) front-view of the droplet 
values and the peak voltage values required to jet a droplet out of the $120 \mu \mathrm{m}$ inkjet nozzle. As the viscosity of the ink increases, the voltage necessary to eject the droplet increases. This results in a larger droplet as well as a larger ejection speed. The average droplet diameter and droplet ejection speed for each ink was used to calculate relevant non-dimensional numbers, viz., Reynolds number, Weber number, Ohnesorge number, and Capillary number, i.e., the ratio of Weber number to the Reynolds number (Table 1). The numbers clearly show that all four inks fall in Region I (Fig. 2a) as defined by Schiaffino and Sonin [20].

\subsection{Aligned fiber mats}

Nylon 6 was chosen as the material for the fiber mats, given its prior demonstrated use in 3D printed FrSCs [9]. Three different aligned Nylon 6 fiber mats of varying effective area coverage (EAC) values were produced using the near-field direct-write setup described in Spackman et al. [9]. Figures 4a-c depict the characteristic structure of these aligned mats. The fiber diameters were seen to have a log-normal distribution with a mean of $\sim 1.34 \mu \mathrm{m}$ and a standard deviation of $0.562 \mu \mathrm{m}$ [33]. The overall size of the mats was $15 \mathrm{~mm}$ X $15 \mathrm{~mm}$. Figures 4a-c show that an increase in the EAC value correlates directly to an increase in the fiber number density of the mat. Therefore, these mats with EACs of $40 \%, 60 \%$ and $80 \%$ will be referred to as the low-density (LD), mediumdensity (MD) and high-density (HD) mat, respectively for the remainder of this paper. The average spacing between the fibers (denoted by $w$ in Fig. 4 ) was seen to be $4 \mu \mathrm{m}, 2.3 \mu \mathrm{m}$, and $0.6 \mu \mathrm{m}$, respectively, for the LD, MD, and HD mats. The fibers in the HD mat were seen to have more lateral attachments with the neighboring fibers as indicated by arrows in Fig. 4c.

\begin{tabular}{|c|c|c|c|c|}
\hline \multicolumn{5}{|c|}{ Table 1: Ink selection for high-speed imaging study } \\
\hline & Ink 1 & Ink 2 & Ink 3 & Ink 4 \\
\hline Vol. \% Glycerol & 0 & 20 & 50 & 80 \\
\hline Vol.\% DI Water & 100 & 80 & 50 & 20 \\
\hline Viscosity $\left(\mathrm{Pa}^{*}\right)^{1}$ & 0.0009 & 0.0016 & 0.0069 & 0.0669 \\
\hline Density $\left(\mathrm{kg} / \mathrm{m}^{3}\right)^{2}$ & 996.9 & 1060.2 & 1144.2 & 1217.1 \\
\hline $\begin{array}{l}\text { Surface Tension } \\
\left(\mathrm{dyn} / \mathrm{cm}^{2}\right)\end{array}$ & 72 & 70 & 68 & 65 \\
\hline Voltage & $17 \mathrm{~V}$ & $25 \mathrm{~V}$ & $40 \mathrm{~V}$ & $75 \mathrm{~V}$ \\
\hline $\begin{array}{l}\text { Average Droplet } \\
\text { Diameter }(\mu \mathrm{m})\end{array}$ & 98 & 108 & 128 & 152 \\
\hline $\begin{array}{l}\text { Average Ejection } \\
\text { Speed }(\mathrm{m} / \mathrm{s})\end{array}$ & 0.962 & 3.02 & 2.54 & 6.31 \\
\hline Reynolds Number & 175 & 176 & 53.9 & 19.2 \\
\hline Weber Number & 2.10 & 12.2 & 13.9 & 125 \\
\hline $\begin{array}{l}\text { Ohnesorge } \\
\text { Number }\end{array}$ & 0.008 & 0.019 & 0.069 & 0.581 \\
\hline $\begin{array}{l}\text { Capillary } \\
\text { Number } \\
\end{array}$ & 0.012 & 0.069 & 0.258 & 6.51 \\
\hline Room Temp. & \multicolumn{4}{|c|}{$25^{\circ} \mathrm{C}$} \\
\hline${ }^{1}$ From Ref. [31] & \multicolumn{4}{|c|}{${ }^{2}$ From Ref. [32] } \\
\hline
\end{tabular}

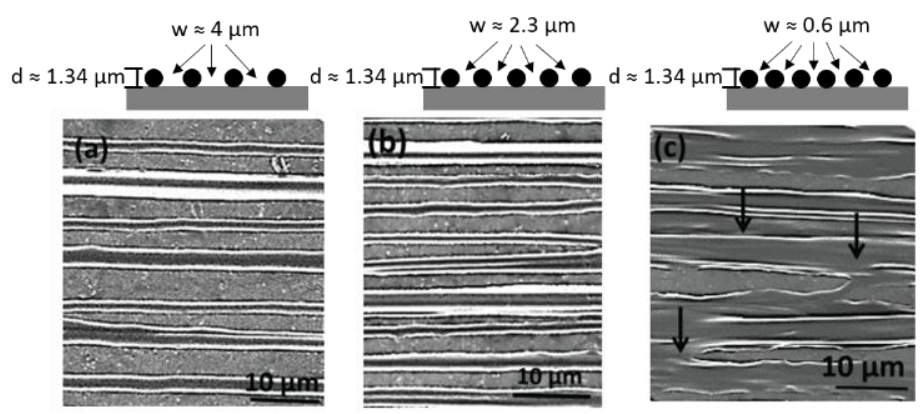

Fig. 4: Aligned fiber mats (a) EAC $40 \%$, low-density mat (b) EAC $60 \%$, medium-density mat (c) EAC $80 \%$, high-density mat

\subsection{Substrate}

The substrate used for the high-speed imaging study has to mimic the surface encountered by the droplet during the 3D printing operation (Fig. 3b-c). To do this, glass slides were first coated with a thin uniform layer of TangoPlus FLX930. This polymer layer was then cured. The fiber mats were subsequently stamped onto this UV cured polymer layer to mimic the surface encountered by the droplet during the 3D printing operation (Fig. 1). This glass slide coated with the UV curable polymer and fibers was then placed under the inkjet nozzle (Fig.3a) to be used as the substrate for the high-speed imaging study. The substrate was mounted such that the aligned fibers were oriented along the $\mathrm{X}$-axis of the 3D printer (Figs. 3a-c). In addition to the LD, MD and HD fiber substrates, a plain polymer substrate (without the fibers) was also prepared to generate the baseline spreading data. 


\subsection{Test conditions}

Two different tests were conducted using the high-speed imaging setup. The first test involved the study of single droplets impinging on stationary and moving substrates. The second set of tests involved the study of overlapping droplets that were deposited in the burst-mode to generate lines on the substrate. Five replicates were conducted for each of the ink-substrate combinations. Both the front-view and the top-view of the droplets/lines were captured at a frame-rate of 11000 frames/second, using the high-speed camera. A digital image analysis program was then used in Matlab $^{\mathrm{TM}}$ to post-process the images and extract the relevant quantitative metrics. The results from these studies are presented in Sections 4-6.

\section{Single Droplet Impingement Study: Stationary Substrate}

There are three droplet shape parameters that are of interest in these studies (Figs. 3b-c): 1) $\mathrm{D}_{\perp}$ : Diameter of the droplet

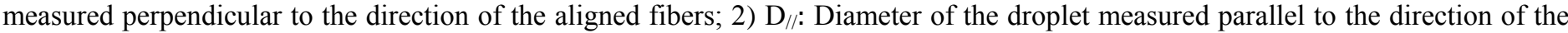
aligned fibers; and 3) $\mathrm{H}$ : Height of the droplet. $\mathrm{D}_{/ /}$and $\mathrm{H}$ can be measured only in the top-view and front-view, respectively (Figs. 3bc), while $\mathrm{D}_{\perp}$ is the measurement common to both views. In order to compare data from multiple replicates, the droplet shape parameters are non-dimensionalized by dividing by the original diameter of the droplet ejected by the inkjet nozzle $\left(\mathrm{D}_{\mathrm{o}}\right)$, similar to the spreading factor $\beta$ presented in Fig. $2 b$.

The black markers in Figs. 5a-b show the typical time-dependent variations seen in the spreading factors $\left(\mathrm{D}_{\perp} / \mathrm{D}_{\mathrm{o}}\right)$ and $\left(\mathrm{D}_{/ /} / \mathrm{D}_{\mathrm{o}}\right)$ for Inks 1 and 4 when deposited on a high-density fibrous substrate. The comparative data for the plain substrate is overlaid in grey to
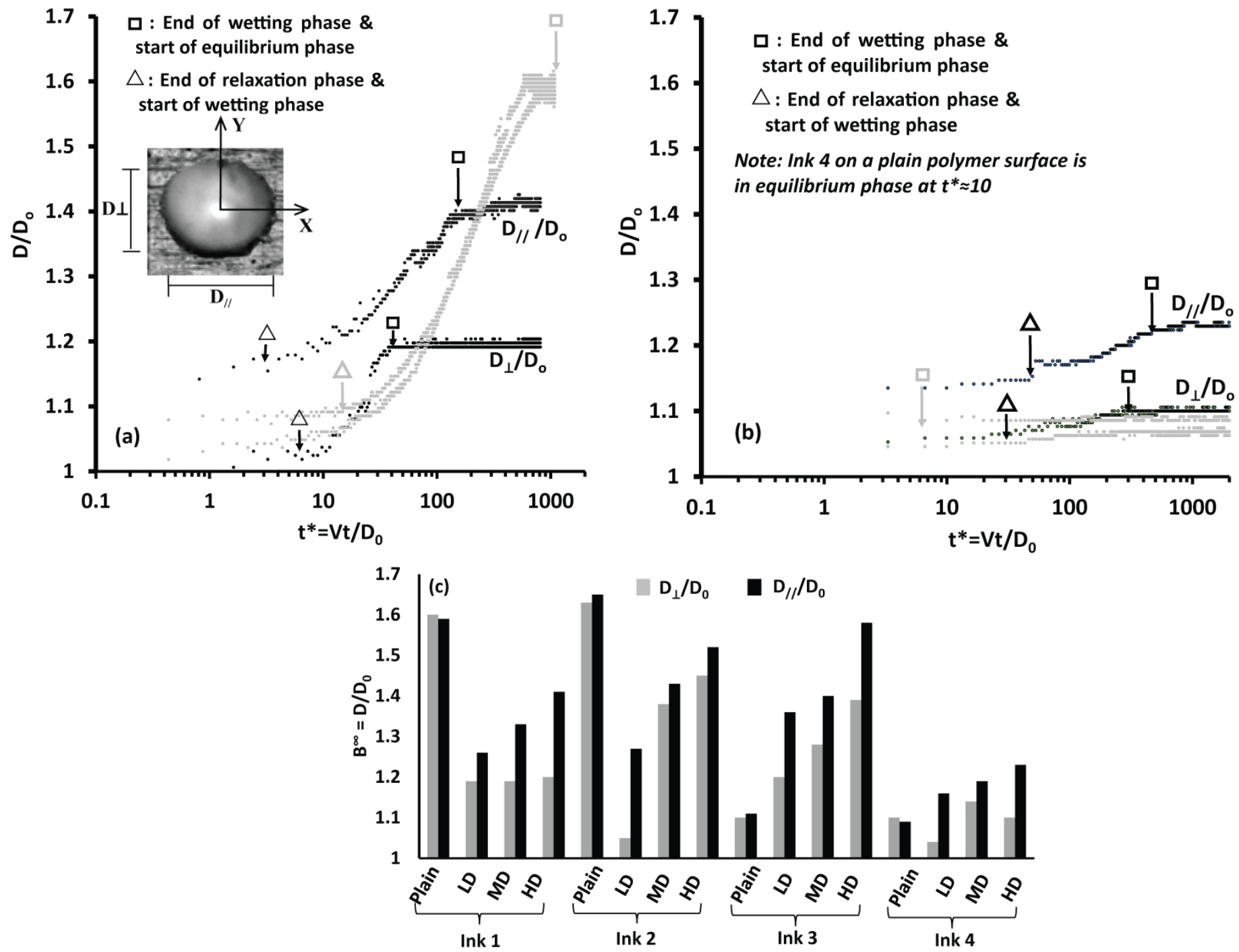

Fig. 5: Evolution of $\left(D_{\perp} / D_{0}\right)$ and $\left(D_{/ /} / D_{o}\right)$ (a) Ink 1 droplet deposited on a stationary HD fiber substrate (black) and a plain polymer substrate (grey) (b) Ink 4 droplet deposited on a stationary HD fiber substrate (black) and a plain polymer substrate (grey) (c) Steady-state diameter data for every ink-substrate combination 
highlight the effect of the fibrous substrate on Inks 1 and 4. The horizontal axis in Figs. 5a-b is plotted in log-scale and it represents the non-dimensional time $t^{*}$ (refer Section 2.2) after the droplet has contacted the substrate. As seen in Figs. 5a-b, a majority of the data points collected in this study are in the region of $t^{*}>1$, confirming that the spreading observed in these experiments is in the wetting/equilibrium phase as defined by Rioboo et. al [21] in Fig. $2 b$.

Figure $5 \mathrm{c}$ shows the values of the equilibrium spreading factors of the droplet for each of the ink-substrate combinations. There are three main trends observed in the data:

1. Droplet spreading on plain substrates can be divided into two categories:

- Low viscosity inks (Inks 1 and 2) that spread for a longer duration of $\sim 160 \mathrm{~ms}$ (which is the time in between the triangle and square shaped markers in Fig. 5a). This longer duration of spreading also results in those inks having relatively large spreading factors of $\sim 1.6$ at equilibrium (Fig. $5 \mathrm{c}$ ); and

- High viscosity inks (Inks 3 and 4) that spread only for a short duration of $\sim 1 \mathrm{~ms}$, thereby resulting in a smaller spreading factor of 1.1 at equilibrium.

2. For all ink and fibrous substrate combinations, the $\left(\mathrm{D}_{/ /} / \mathrm{D}_{\mathrm{o}}\right)$ values are seen to be consistently greater than the corresponding $\left(D_{\perp} / D_{o}\right)$ values, indicating that the droplet is preferentially spreading along the length of the fibers. This indicates that the droplets encountered during 3D printing of FrSCs are semi-ellipsoidal in shape as opposed to the semi-spheroidal droplets encountered on the plain substrate. The equilibrium spreading factors $\left(D_{\perp} / D_{0}\right)$ and $\left(D_{/ /} / D_{0}\right)$ are also seen to increase with an increase in the fiber density of the mat.

3. In all substrate scenarios, the ink with the highest viscosity (Ink 4) spreads the least when compared to the other inks.

The height data collected for single droplets on stationary substrates is not included here for brevity. However, given the conservation of droplet volume, the general trends in the height data can be inferred from Fig. 5c, with the height decreasing with an increase in the spreading factors.

\subsection{Spreading on plain polymer substrates}

During the wetting phase of a droplet on a plain substrate, the viscous friction within the droplet competes with capillarity [23]. The work of Jung et al. [34] showed that for cases when the surface energy is the driving force, the evolution of the spreading factor is expected to follow Tanner's law given by $\alpha\left[\mathrm{t}^{*}\right]^{\mathrm{P}}$, where $\alpha$ is a constant, $\mathrm{t}^{*}$ is dimensionless time, and the exponent $\beta$ is $\sim 0.1$. The data points for Ink 1 result in $\alpha$ and $\beta$ values of 0.8219 and 0.0973 , respectively. For Ink 2 the values of $\alpha$ and $\beta$ are 0.8404 and 0.104 , respectively. This agreement with the Tanner's law indicates that for low-viscosity Inks 1 and 2, the spreading is primarily driven by surface energy, resulting in a large spreading factor of $\sim 1.6$ at equilibrium.

As the viscosity of the ink increases, the viscous dissipation within the droplet increases, thereby slowing the spread of a droplet after its impact [35]. The equation for the conservation of energy of the droplet is given by $[30,36]$

$$
\mathrm{KE}_{1}+\mathrm{SE}_{1}=\mathrm{SE}_{2}+\mathrm{E}_{\mathrm{v}} \text {, [Eq. 1] }
$$

where $\mathrm{KE}_{1}$ and $\mathrm{SE}_{1}$ represent the kinetic energy and surface energy of the droplet, respectively, before impact. The surface energy of the droplet after impact and the subsequent viscous dissipation in the droplet are denoted by $\mathrm{SE}_{2}$ and $\mathrm{E}_{\mathrm{v}}$, respectively. The higher viscosities of Inks 3 and 4, necessitate higher ejection voltages implying that the kinetic energy of these droplets before impact is slightly higher than those for Inks 1 and 2 (Table 1). However, for these high viscosity inks the viscous dissipation term $\mathrm{E}_{\mathrm{v}}$ will dominate after impact. This implies that there exists a critical viscosity value for which the viscous dissipation term on the right hand side of Eq. 1 grows large enough to dominate the spreading behavior after impact. In the case of the plain substrate, this change in spreading behavior from a surface energy dominated to a viscous dissipation dominated regime is seen between Inks 2 and 3 , i.e., between viscosity values of $0.0016-0.0069 \mathrm{~Pa}^{*} \mathrm{~s}$ (Fig. 5c). This matches the range of viscosity values identified by Chen et al. [37] for a similar transition seen during their inkjet-based droplet impact studies. The effect of increased viscosity is the highest for Ink 4 as indicated by its Capillary number being $>1$ (Table 1), which implies that the viscous forces are dominant [38].

\subsection{Spreading on fibrous substrates}

For fibrous substrates, the $\left(D_{/ /} / D_{0}\right)$ values encountered in the wetting/equilibrium phase are seen to be consistently greater than the corresponding $\left(D_{\perp} / D_{o}\right)$ values. As seen from Figs. 5a-b, this trend is even visible for time domains where $1<t^{*}<10$, which indicates that the presence of fibers is likely promoting the preferential mode of spreading even during the early impact phases of the droplet (Fig. 2b). This observed spreading behavior is similar to the trends seen in the studies involving droplet impact on microgrooved substrates [18].

The extent of preferential spreading is seen to increase for all inks with an increase in the fiber mat density. There are two factors that influence this trend, viz., the effect of the surface energy of the Nylon 6 fibers and the wicking of the droplet by the fibrous mat. The equilibrium contact angle of the four inks was measured to be $\sim 80^{\circ}$ on the plain polymer substrate and is known to be $\sim 62^{\circ}$ on bulk Nylon 6 [39]. Given the lower contact angle on Nylon 6, it is expected that the presence of Nylon 6 fibers on the polymer substrate will promote droplet spreading, as evidenced by an increase in both $\left(D_{/ /} / D_{0}\right)$ and $\left(D_{\perp} / D_{0}\right)$ values in Fig. 5c. The preferential spreading along the length of the fibers can be explained by the wicking caused by the spaces in between the individual fibers [22]. If the void space between the fibers can be represented as a channel of depth $d$, equal to the diameter of the fiber, and width $w$, equal to 
the spacing between the fibers, then the angle of wetting that the liquid experiences in the channel has a critical value, $\theta_{\text {critical }}$, defined as [22]

$$
\cos \theta_{\text {critical }}=\mathrm{w} /(2 \mathrm{~d}+\mathrm{w}) \quad \text {. [Eq. 2] }
$$

As the value of $\theta_{\text {critical }}$ goes to zero, the surface acts as a plain substrate, but as the value of $\theta_{\text {critical }}$ goes to $90^{\circ}$, the substrate acts as a classical porous medium [22] that preferentially wicks the droplet. Based on the average diameter and spacing of the fibers in Fig. 4, the $\theta_{\text {critical }}$ values of LD, MD, and $\mathrm{HD}$ mats are identified to be $47.3^{\circ}, 51.3^{\circ}$, and $56.3^{\circ}$, respectively. This increase in the critical wetting angle experienced by the liquid with an increase in the number density of the mat explains the trend seen in the $\left(D_{/ /} / D_{o}\right)$ spreading factor.

\subsection{Effect of occasional micro-scale surface roughness variations}

The fibrous substrates used for the single-droplet impingement study were occasionally observed to have local variations in the surface roughness characteristics of the mats. These variations are primarily attributed to the stochasticity in the drum-based nearfield electrospinning process used to manufacture the mats [33]. While these micro-scale surface roughness variations only constituted $<1.5 \%$ of the overall $15 \mathrm{~mm} \mathrm{X} 15 \mathrm{~mm}$ area of the mats, they were seen to result in interesting local droplet spreading characteristics. Two extreme examples of these variations are presented below. The first case involves the presence of fiber bundles in a low-density fiber mat, and the second case involves the presence of voids in a high-density fiber mat.

\subsubsection{Effect of fiber bundles in low-density mats}

The diameter of the occasional fiber bundles in the case of the low-density mats was seen to follow a logistic distribution with a mean of $9.389 \mu \mathrm{m}$ and a standard deviation of $3.08 \mu \mathrm{m}$. These fiber bundles, which are $\sim 7$ times the diameter of the individual fibers themselves, cover only $\sim 1.5 \%$ of the total area of the low-density mats. While the average spacing of these fiber bundles is $\sim 650 \mu \mathrm{m}$, Fig. 6 discusses a rare case when two fiber bundles are close enough to influence a single droplet. Figure 6a shows the characteristic $\left(\mathrm{D}_{\perp} / \mathrm{D}_{\mathrm{o}}\right)$ and $\left(\mathrm{H} / \mathrm{D}_{\mathrm{o}}\right)$ curves for a droplet deposited on a low-density fiber mat that shows the presence of two fiber bundles separated by a distance of $\sim 100 \mu \mathrm{m}$. The droplet lands in between two fiber bundles and starts to spread. It is immediately pinned on its side by the two fiber bundles, because of which the $\left(\mathrm{D}_{\perp} / \mathrm{D}_{\mathrm{o}}\right)$ value stays at a steady-state value of 1.2 for $\sim 18 \mathrm{~ms}$. During this time the height of the droplet is continuously decreasing, which implies that the droplet continues to spread along the length of the fibers due to the wicking effect (refer Section 4.2), while being pinned in the orthogonal direction. This phenomenon is shown pictorially in Fig. $6 \mathrm{~b}$, where the energy required for the progression of the contact line along the paths $a-b-c-d$ and $e-f-g-h$ (orthogonal to the fiber bundles) is greater than that energy required for the droplet to spread along the length of the fiber. The surface energy barrier posed by the two fiber bundles is overcome after $\sim 18 \mathrm{~ms}$. The spreading

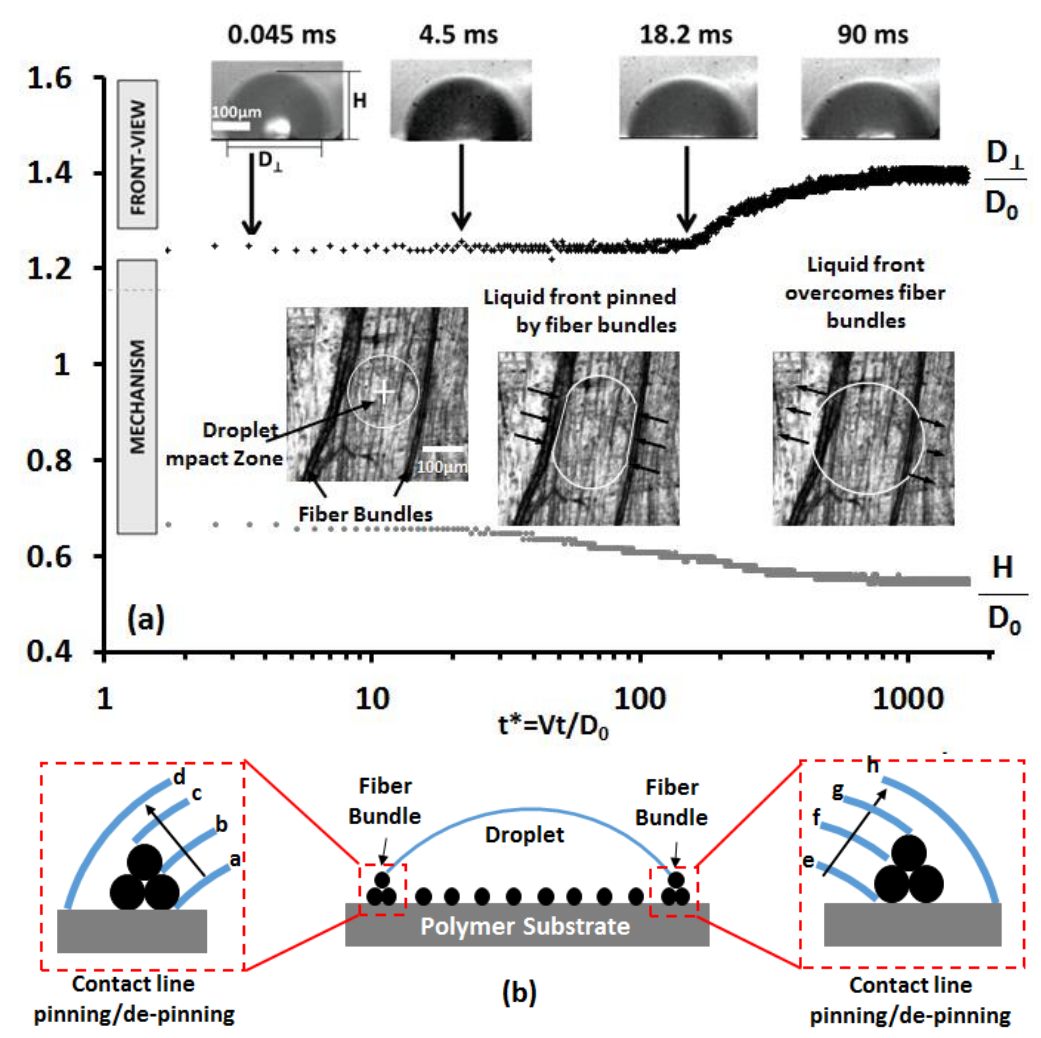

Fig. 6: The effect of fiber bundles on the droplet contact line (Ink 2 on LD Substrate)

then proceeds along both the $\mathrm{D}_{\perp}$ and $\mathrm{D}_{/ /}$directions. The droplet then has a new steady state $\left(\mathrm{D}_{\perp} / \mathrm{D}_{\mathrm{o}}\right)$ value of $\sim 1.4$ after $\sim 90 \mathrm{~ms}$ (Fig. 6a).

\subsubsection{Effect of voids in high-density mats}

The width of the voids on the high-density mats (dimension "W" in Fig. 7) was seen to follow a logistic distribution with a mean of $11.8 \mu \mathrm{m}$ and a standard deviation of $4.05 \mu \mathrm{m}$. These voids, which have a width $\sim 20$ times the spacing between the individual fibers, comprise only $\sim 0.75 \%$ of the area of the high-density mats. While the average spacing of these voids (dimension "S" in Fig. 7) is $\sim 1170 \mu \mathrm{m}$, Fig. 7 discusses a rare case when two voids are close enough to influence a single droplet. Figure $7 \mathrm{a}$ shows the characteristic $\left(\mathrm{D}_{\perp} / \mathrm{D}_{\mathrm{o}}\right)$ and $\left(\mathrm{D}_{/ /} / \mathrm{D}_{\mathrm{o}}\right)$ curves for an Ink 4 droplet deposited at a spot in the high-density mat that is sandwiched between two voids spaced at $\sim 120 \mu \mathrm{m}$. In a high-density mat most of the substrate is covered with fibers with high surface energy. Therefore, 
any presence of void patches implies a local decrease in the surface energy value that will affect the spreading characteristics. This is seen in the form of the droplet spreading for $\sim 1.7 \mathrm{~ms}$, when one edge of the droplet meets a void space and gets pinned. At $\sim 8.7 \mathrm{~ms}$, both edges affecting the $\left(\mathrm{D}_{\perp} / \mathrm{D}_{\mathrm{o}}\right)$ measurement are pinned by the voids on the two sides of the droplet (Fig. 7b). After this the fibers are seen to promote spreading along their length due to the wicking effect, which results in an increase in the $\left(D_{/ /} / D_{0}\right)$ diameter for the next $\sim 25 \mathrm{~ms}$ before it reaches a steady-state value of 1.68. Given this mode of preferential spreading along the length of the fibers, a high level of eccentricity is observed in the droplet. This is indicated by the $125 \%$ difference between the $\left(D_{\perp} / \mathrm{D}_{\mathrm{o}}\right)$ and $\left(\mathrm{D}_{/ /} / \mathrm{D}_{\mathrm{o}}\right)$ values at steady-state, which is significantly higher than any of the cases seen in Fig. 5.

The above two cases are variations of the pinning/de-pinning of the three-phase contact line observed in studies involving impact of droplets on grooved substrates [18]. While the percentage of the overall fiber mat affected by such micro-scale surface roughness variations is negligible, these variations do result in interesting local droplet behaviors that should be accounted for, especially in cases involving printing of single droplets over those areas.

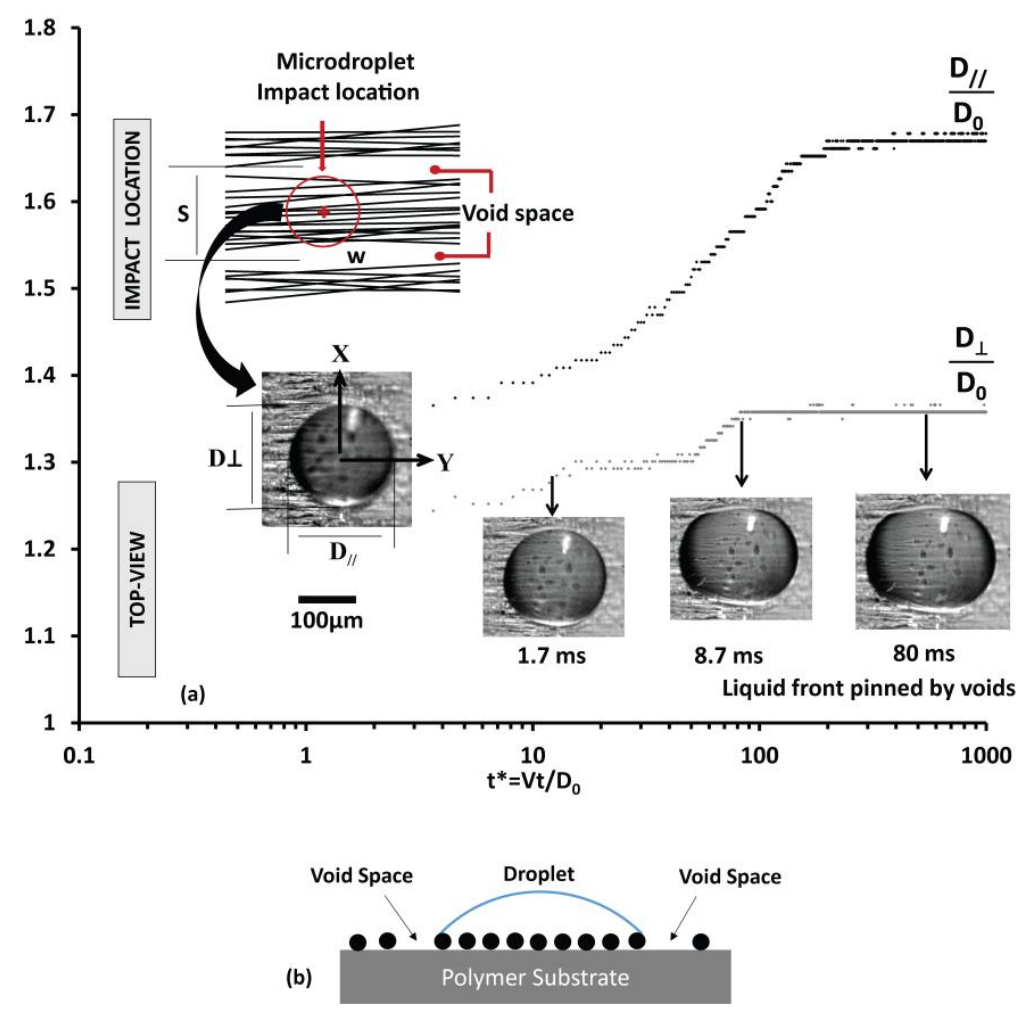

Fig. 7: The effect of void space on the droplet contact line (Ink 4 on HD Substrate)

\section{Single Droplet Impingement Study: Moving Substrate}

In order to study the effect of the substrate velocity on the droplet spreading mechanisms, a moving substrate study was performed. In this study, the front-view of the droplet (Fig. 2c) was imaged using the high-speed camera while the substrate was moved at a constant speed of $10 \mathrm{~mm} / \mathrm{s}$, a speed commonly used for FrSC 3D printing applications [9]. Two different tests were performed for each of the fiber-ink combinations, viz., the in-line printing test and the orthogonal printing test. In the in-line printing tests, the direction of the printing velocity is parallel to the direction of the fiber alignment, whereas in the orthogonal printing tests the direction of the printing velocity is perpendicular to the fiber alignment. Figure $8 \mathrm{a}$ and $8 \mathrm{~b}$ show the orientations of these two tests.

In the front-view, the height $(\mathrm{H})$ of the droplet is measured along with the diameter parallel to the imparted velocity $\left(\mathrm{D}_{/ / \text {-Vel. }}\right)$. Both of these parameters are critical for 3D printing because they dictate the height of the printed layer as well as the extent of overlap needed between successive droplets while printing a line. For the in-line printing case (Fig. 8a), $\mathrm{D}_{/ / \text {-vel. will be equivalent to }}$ $\mathrm{D}_{/ /}$(defined in Section 4), whereas for the orthogonal printing scenario (Fig. 8b), $\mathrm{D}_{/ / \text {-vel. }}$ will be equivalent to $\mathrm{D}_{\perp}$ (defined in Section 4). Figure $8 c$ overlays the characteristic $\left(\mathrm{D}_{/ /-\mathrm{vel}} / \mathrm{D}_{\mathrm{o}}\right)$ and $\left(\mathrm{H} / \mathrm{D}_{\mathrm{o}}\right)$ data for Ink 4 during the orthogonal and in-line printing scenarios, on a HD mat. When compared to the stationary substrate data in Fig. 5c it is clear that the addition of the velocity acts as an external perturbation that influences both the wicking along the length of the fibers during in-line printing, and the pinning/de-pinning of the three-phase contact line seen during orthogonal printing. The effect of the perturbation is more pronounced during in-line printing as seen by the increased value of the steady state $\left(\mathrm{D}_{/ /-\mathrm{Vel}} / \mathrm{D}_{\mathrm{o}}\right)$. 
Figure 9 shows the steady-state $\left(\mathrm{D}_{/ /-\mathrm{Vel}} / \mathrm{D}_{\mathrm{o}}\right)$ and $\left(\mathrm{H} / \mathrm{D}_{\mathrm{o}}\right)$ values for all the ink-substrate combinations, while printing in the orthogonal and in-line modes. Across the entire data set, the steady-state $\left(\mathrm{D}_{/ /-\mathrm{vel}} / \mathrm{D}_{\mathrm{o}}\right)$ values are observed to be higher for the in-line printing tests than for the orthogonal printing tests. In general, the steady-state $\left(H / \mathrm{D}_{\mathrm{o}}\right)$ values are higher for the orthogonal printing tests than for the in-line printing tests. The data suggests that in order to get a higher height of the printed droplet, it may be advantageous to have the print velocity be perpendicular to the direction of fiber alignment.

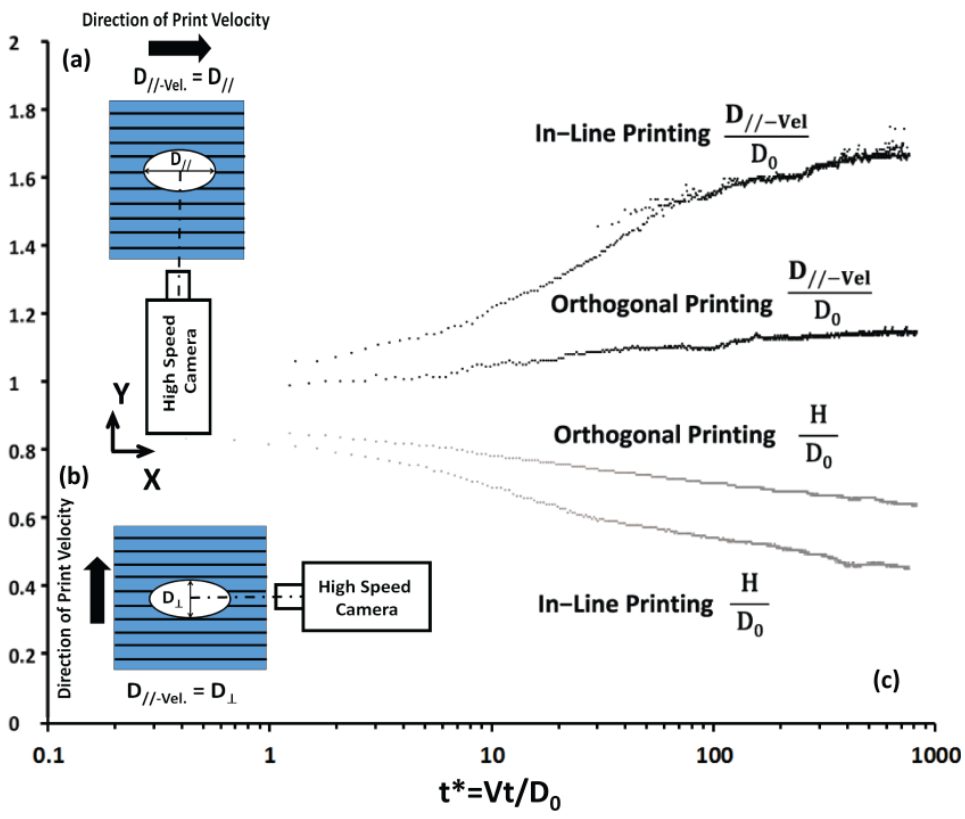

Fig. 8: Effect of substrate velocity on Ink 4 droplet spreading over HD fiber substrate. (a) In-line printing setup (b) Orthogonal printing setup (c) comparison of the in-line and orthogonal printing of Ink 4 on HD mat (Note: The horizontal axis is in logarithmic scale)

\section{Orthogonal and In-line Printing of Lines}

The moving substrate study was extended to the printing of lines on the fibrous substrates. This study was conducted to shed light on the cumulative effect of a series of droplets, on the dimensions of a printed line. For this experiment, droplets were printed from the inkjet nozzle at a frequency of $100 \mathrm{~Hz}$. Both orthogonal and in-line printing modes were investigated at a printing velocity of $10 \mathrm{~mm} / \mathrm{s}$. These conditions resulted in a $\sim 50 \%$ overlap between consecutive droplets.

The effective line width resulting from a series of overlapping droplets depends on the initial diameter of the original droplet, the extent of overlap with the deposited liquid line front, and the surface energy of the substrate. Figure 10 shows an illustration of the phenomenon that occurs while printing a line. The location of the droplet landing on the substrate is given by A-B-C-D (Fig. 10).

As seen, this droplet is positioned such that it has overlap with the liquid line front being printed. As soon as the droplet lands on the substrate, the surface tension of the liquid causes the leading edge of the droplet to be drawn in, as depicted by the arrows in Fig. 10. This causes the leading edge of the line to recede from A-B-C to E-F-G.

Figure 11 shows the above mentioned mechanism played out while printing a line on a plain polymer substrate and a highdensity mat. The time-duration of $10 \mathrm{~ms}$ shown here is the time between successive droplet depositions at the printing frequency of $100 \mathrm{~Hz}$. Figures 11a-d show the receding phenomenon seen while printing on the plain polymer substrate. For the orthogonal printing mode (Figs. 11e-h), where the printing velocity is perpendicular to the direction of fiber alignment, one sees an arrest of this receding front in the last frame due to pinning. In-line printing on the same mat is seen to further aid this receding of the leading edge (Figs. 11i-1). This data once again confirms the wicking phenomenon occurring along the length of the fibers. 


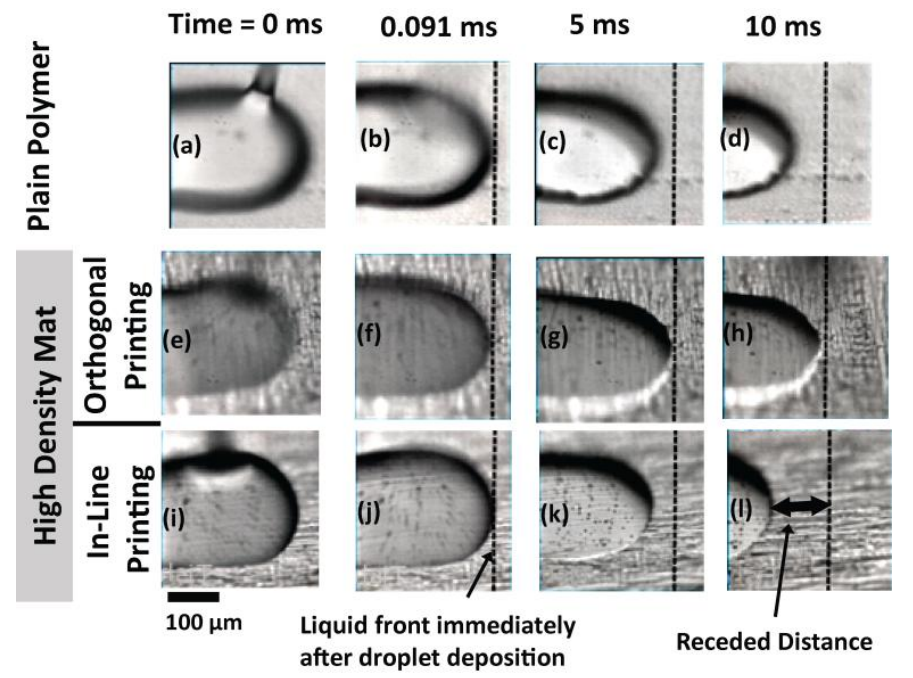

Fig. 11: Evidence of the edge receding phenomenon seen on substrates while printing with Ink 4 (Note: The dotted line denoting the liquid front at $0.091 \mathrm{~ms}$ is at the same location in all images)

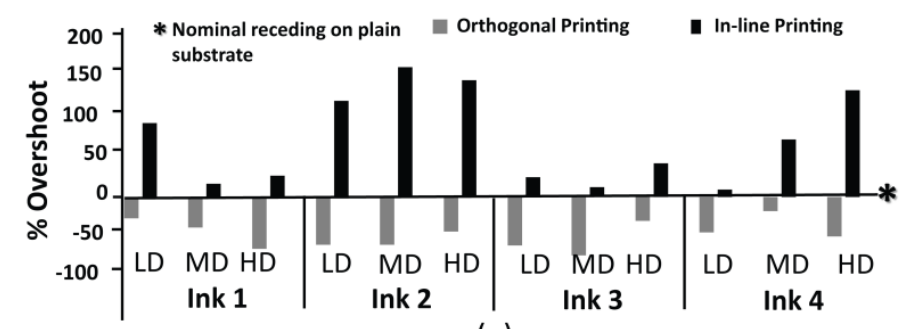

(a)

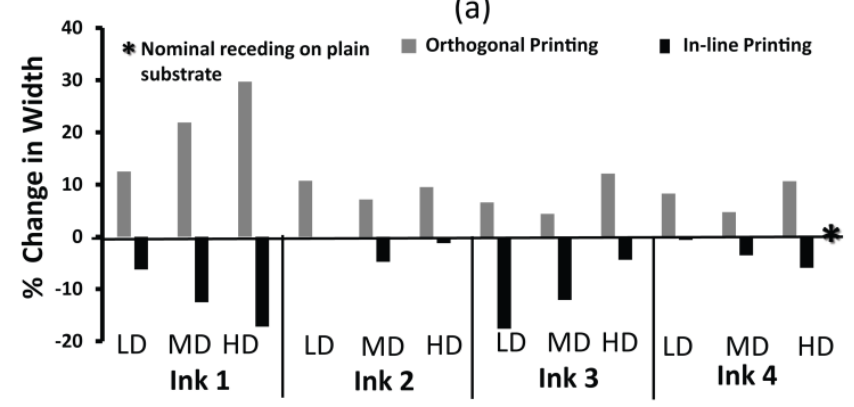

(b)

Fig. 12: Effect of fibrous substrates on line printing (a) leading edge retraction (b) line width

$\mathrm{D}_{\perp}$ and $\mathrm{D}_{/ /}$diameters is seen to be $51 \mu \mathrm{m}$ for orthogonal printing (Fig. 13g) and $96 \mu \mathrm{m}$ for in-line printing (Fig. $13 \mathrm{~m}$ ), confirming the similarities between this UV curable polymer and Ink 4.

The line printing studies reveal the effect of varied lateral spacing, on the width of the resulting line artifacts. It is interesting to note that for the in-line printing scenario, a clear distinction between the two line edges is seen even at a low spacing of $2.4 \mathrm{D}_{\mathrm{o}}\left(\mathrm{Fig}_{\mathrm{g}}\right.$. 13o). The plain polymer substrate provides the next best lateral resolution with the distinction between the two line edges appearing around a spacing of $2.7 \mathrm{D}_{\mathrm{o}}$ (Fig. 13d). Orthogonal printing provides the worst lateral resolution with the line edges becoming visible only at the largest spacing of $3.2 \mathrm{D}_{\mathrm{o}}$ (Fig. 131). The line width measured at the largest spacing of $3.2 \mathrm{D}_{\mathrm{o}}$ is $350 \mu \mathrm{m}$ for the plain polymer substrate. The corresponding values are 380 and $300 \mu \mathrm{m}$, respectively, for the orthogonal and in-line printing cases. These findings are indicative of the trends seen in the width of the lines printed during the high-speed imaging study (Refer Sec. 6).

The UV cured line artifacts show an edge roughness effect that is not observed in the high-speed imaging experiments. Figure 14a shows a close up of Fig. 13h, which is the case of orthogonal line printing on a low-density mat. Here the UV curable ink is seen to spread perpendicular to the nominal line edge (dotted line in Fig. 14a) due to the presence of fibers. This effect is accentuated after the UV curing step, leading to increased edge roughness of the line artifact.

Figure $12 \mathrm{a}$ shows the percentage overshoot in the length of the line printed on fibrous substrates when compared against that seen for the plain polymer substrate, for all percentage overshoot implies that the line has retracted more than the baseline polymer case $(*$ in Fig. 12a), whereas a negative value indicates that the line has receded less than the of all number densities is seen to reduce the extent of retraction, whereas in-line printing across all mat densities is seen to aid it. Figure 12b depicts the width of the lines printed for all the ink/substrate combinations. Orthogonal printing is seen to result in wider line-widths than in-line
printing, for all inks and fiber number densities. This again points to the same phenomenon seen while printing droplets, that the droplets prefer to spread along the length of the

\section{Translation of Findings to 3D Printing with UV \\ Curable Ink}

While UV curable inks cannot be used to confirm the high-speed imaging results seen during the printing of single droplets and lines, the steady-state effects of the spreading can be confirmed after the ink has been printed and cured. Fis validation study, TangoPlus FLX930, a UV curable density mat. This UV curable polymer mimics Ink 4 in its surface tension and viscosity values [40]. The mat regions chosen for this study were selected to avoid the variations seen in Sec. 4.3. Both single droplet and line printing studies were conducted on the substrate moving at $10 \mathrm{~mm} / \mathrm{s}$. For the ranging from $2.2 \mathrm{D}_{\mathrm{o}}$ to $3.2 \mathrm{D}_{\mathrm{o}}$ were printed to study the effects of lateral spreading. The printed artifacts were UV cured and hen inspected under a microscope.

printed on a plain polymer substrate and a low-density fiber mat. Both orthogonal (Figs. 13g-l) and in-line (Figs. 13 m-r) printing modes are used on the fiber mat. For both the orthogonal and in-line printing scenarios, the diameter of the deposited droplet is seen to be larger along the length of the fibers (Figs. 13a, 13g, and 13m). The difference between the

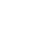




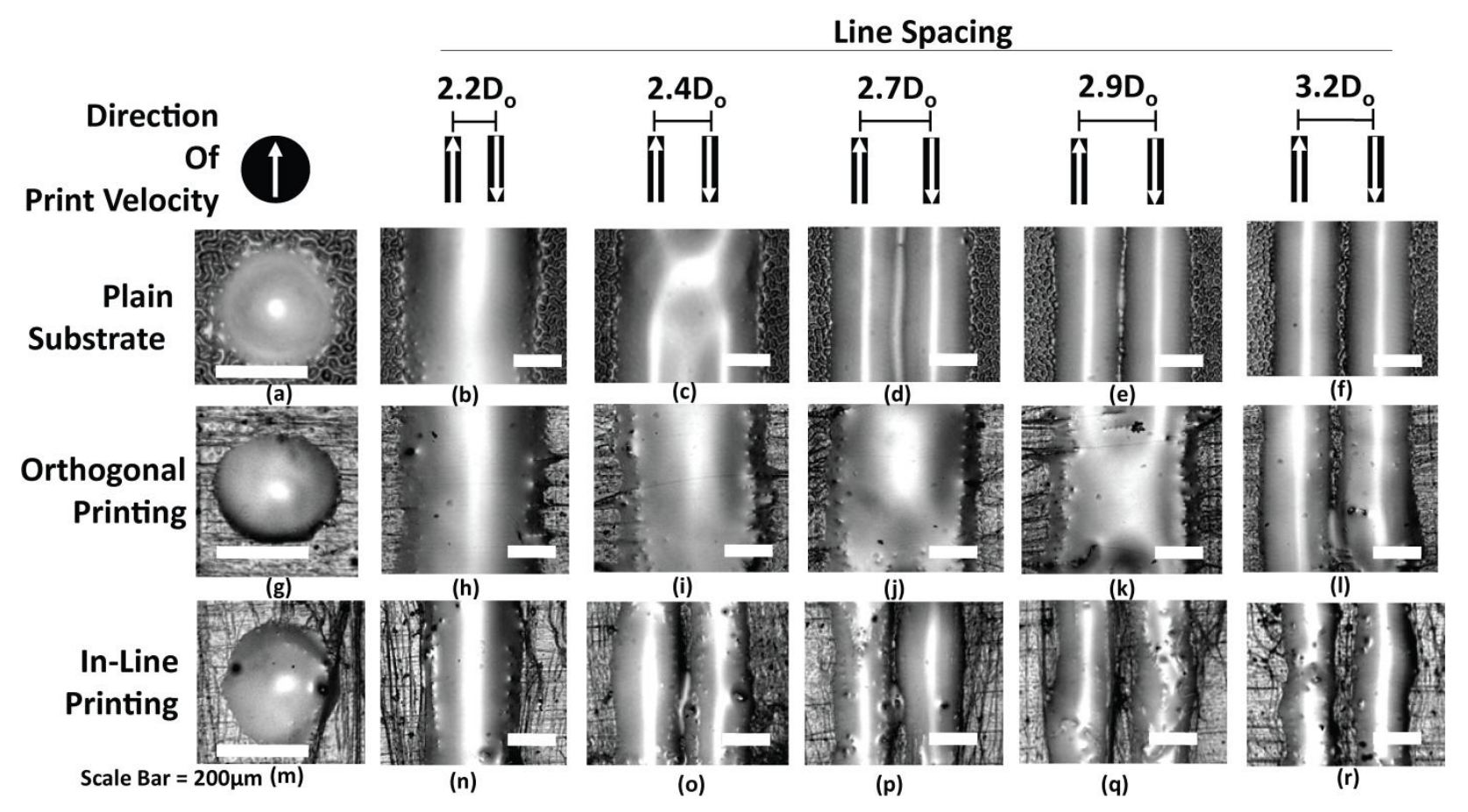

Fig. 13: 3D printed artifacts with UV curable ink

The edge roughness (ER) of the line artifact can be quantified as, $E R=\frac{1}{n} \sum_{i=1}^{n}\left|y_{i}\right|$, where $n$ represents the number of samples and $\left|y_{i}\right|$ is the absolute value of difference between the nominal line edge and the cured extremities. Figure 14b shows the $\left|y_{i}\right|$ values for the image in Fig. 14a, and Table 2 summarizes the ER results seen for all the cases depicted in Fig. 13. A review of the data in Table 2 shows that the plain polymer substrate has the lowest ER. In general the orthogonal mats have the highest ER, since the ink is wicked along the length of the fibers. However, some in-line printing cases are seen to have high ER values. In those cases (i.e., line spacing of $2.9 \mathrm{D}_{\mathrm{o}}$ and $3.2 \mathrm{D}_{\mathrm{o}}$ ) it is observed that the edge roughness is dictated by nature of the underlying fiber mat (Figs. $13 \mathrm{q}-\mathrm{r}$ ).

Unlike commercial inkjet printers, the FrSC printer developed by Spackman et al. [9] has a significant time delay between the printing and UV curing step. This did not allow for the 3D printing results to capture the phenomenon of the receding droplet train seen in Fig. 11. However, the issue will be relevant for FrSC implementations on commercial-grade inkjet-based 3D printers, where the time-delays are of the order of one or two droplet depositions.

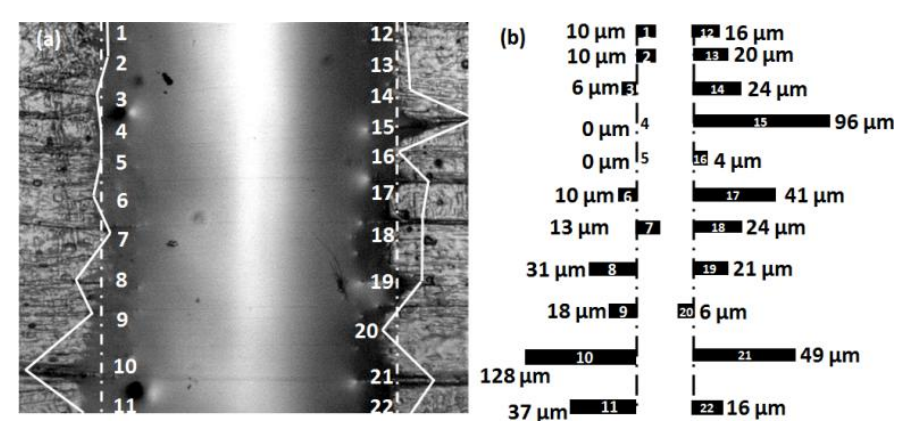

Fig.14: Edge roughness calculation

\begin{tabular}{|c|c|c|c|c|c|}
\hline & \multicolumn{5}{|c|}{ Line Spacing } \\
\hline & $2.2 \mathrm{D}_{0}$ & $2.4 D_{0}$ & $2.7 D_{0}$ & $2.9 D_{0}$ & $3.2 \mathrm{D}_{0}$ \\
\hline $\begin{array}{l}\text { Plain } \\
\text { Substrate ER }\end{array}$ & 9.64 & 3.39 & 8.93 & 12.14 & 10.00 \\
\hline $\begin{array}{l}\text { Orthogonal } \\
\text { Printing ER }\end{array}$ & 26.43 & 31.23 & 28.95 & 24.04 & 21.71 \\
\hline $\begin{array}{l}\text { In-Line } \\
\text { Printing ER }\end{array}$ & 12.32 & 15.18 & 15.71 & 37.68 & 24.29 \\
\hline
\end{tabular}

\section{Future Research Directions}

The findings reported in Section 4-7 pose the following unanswered questions related to the nascent field of 3D printing FrSCs:

- How would the results from the printing of single droplets and lines reported in this paper translate to the printing of a single layer (consisting of overlapping lines)? 
- How would inline and orthogonal printing strategies affect the overall height and edge resolution of the layer?

- How would the above printing strategies influence the overall printing time and dimensional accuracy of the 3D part?

- How would the occurrence of the occasional fiber bundles and voids affect the above findings?

Research efforts directed towards answering the above questions will result in the development of robust process planning and control strategies for 3D printing FrSCs

\section{Conclusions}

The following specific conclusions can be made from this study of droplet interactions on aligned fibrous substrates relevant to the 3D printing of FrSCs:

1. The single droplet impingement studies on stationary substrates reveal that:

- The presence of fibers influences the time taken by the droplet to reach its steady-state shape. Fibrous substrates mitigate the difference in spreading characteristics seen in liquids of differing viscosities.

- The presence of fibers promotes droplet spreading in the direction of alignment of the fibrous mat. Droplets tend to spread more on denser fiber mats due to wicking.

- Droplet spreading characteristics can be affected by rare surface roughness variations in the fiber mats, such as the occurrence of fiber bundles and voids.

2. The droplet impingement studies on moving substrates reveal that:

- The droplets spread the most during in-line printing, i.e., when the direction of the printing velocity coincides with the direction of fiber alignment. The spreading of the droplet is the least during orthogonal printing, i.e., when the direction of the printing velocity is perpendicular to the direction of fiber alignment. This implies that in-line printing will result is a lower layer height as opposed to orthogonal printing.

- The printing of straight lines shows an interesting leading edge retraction phenomenon that gets accentuated the most in the case of in-line printing.

3. The findings of the high-speed imaging studies have been confirmed by 3D printing comparable artifacts using UV curable inks. These printing tests indicate that for a given fiber mat and UV curable ink combination, the choice of the in-line or orthogonal printing strategy has process planning implications for the overall printing time, fiber content, edge resolution and surface quality of the 3D printed FrSC part.

\section{Acknowledgement}

The authors gratefully acknowledge funding support from the US National Science Foundation (Award Number MME 14-62648). The authors would also like to thank MicroFab Inc. for their technical support with the implementation of the inkjet printing system at Rensselaer Polytechnic Institute, NY.

\section{References}

[1] S. Tibbits, 4D Printing: Multi-material Shape Change, Architectural Design, 84(1), (2014) 116-121.

[2] Q. Ge, J.J. Qi, M.L. Dunn, Active Materials by Four-dimension Printing, Applied Physics Letters, 103(13), (2013) 131901 (5 pages).

[3] S. Lapidot, S. Meirovitch, S. Sharon, A. Heyman, D.L. Kaplan, O. Shoseyov, Clues for Biomimetics from Natural Composite Materials, Nanomedicine, 7(9), (2012) 1409-1423.

[4] F. Yan, Y. Liu, H. Chen, F. Zhang, L. Zheng, Q. Hu, A Multi-scale Controlled Tissue Engineering Scaffold Prepared by 3D Printing and NFES Technology, AIP Advances, 4 (3), (2014) 031321 (8 pages).

[5] J. Czyzewski, P. Burzynski, K. Gawe, J. Meisner, Rapid Prototyping of Electrically Conductive Components Using 3D Printing Technology, Journal of Materials Processing Technology, 209(12-13), (2009) 5281-5285. 
[6] C.R. Rocha, T. Perez, R. Angel, D.A. Roberson, C.M. Shemelya, E.W. MacDonald, R.B. Wicker, Novel ABS-based Binary and Ternary Polymer Blends for Material Extrusion 3D Printing, Journal of Materials Research, 29(17), (2014) 1859-1866.

[7] A.J. Lopes, E. MacDonald, R.B. Wicker, Integrating stereolithography and direct print technologies for 3D structural electronics fabrication, Rapid Prototyping Journal, 18(2), (2012) 129-143.

[8] L. Wang, Y. Ma, M. Chen, H. Yao, X. Zheng, W. Yang, An Inkjet Printing Soft Photomask and its Application on Organic Polymer Substrates, Science China: Chemistry, 53(8), (2010) 1695-1704.

[9] C. Spackman, K. Picha, G. Gross, J. Nowak, P.J. Smith, J. Zheng, J. Samuel, S. Mishra, A Novel Multi-material Additive Manufacturing Technique for Fabricating Laminated Polymer Nanocomposite Structures, ASME Journal of Micro and NanoManufacturing, 3(1), (2015) 011008 (11 pages).

[10] A.B.D. Cassie, S. Baxter, Wettability of Porous Surfaces, Transactions of the Faraday Society, v 40, (1944) 546-551.

[11] R.N. Wenzel, Resistance of solid surfaces to wetting by water, Industrial and Engineering Chemistry 28, (1936) 988-994.

[12] W.B. Huang, G.L. Wang, F.Q. Gao, Z.T. Qiao, G. Wang, M.J. Chen, L. Tao, D. Ya, L.F. Sun, Dynamic Surface Wettability of Three-Dimensional Graphene Foam, 23(4), (2014) 046802 (4 pages).

[13] K.P. Hapgood, J.D. Litster, S.R. Biggs, T. Howes, Drop Penetration into Porous Powder Beds, Journal of Colloid and Interface Science 253, (2002) 353-366.

[14] B. Leng, Z. Shao, G. de With, W. Ming, Superoleophobic Cotton Textiles, Langmuir 25, (2009) 2456-2460.

[15] X.F. Wu, Y.A. Dzenis, Droplet on a fiber: geometrical shape and contact angle, Acta Mechanica 185 (2005) 215-225.

[16] S. Protiere, C. Duprat, H.A. Stone, Wetting on two parallel fibers: drop to column transitions, Soft Matter 9, (2013) 271-276.

[17] A. Bedarkar, X.F. Wu, A. Vaynberg, Wetting of liquid droplets on two parallel filaments, Applied Surface Science 256, (2010) $7260-7264$.

[18] J.Y. Chung, J.P. Youngblood, C.M. Stafford, Anisotropic wetting on tunable micro-wrinkled surfaces, Soft Matter 3, (2007) 1163-1169.

[19] L.M. Bellan, H.G. Craighead, Nanomanufacturing Using Electrospinning, Journal of Manufacturing Science and Engineering, 131(3), (2009) 034001, (4 pages).

[20] S. Schiaffino, A.A. Sonin, Molten Droplet Deposition and Solidification at Low Weber Numbers, Physics of Fluids, 9(11), (1997) 3172-3187.

[21] R. Rioboo, M. Marengo, C. Tropea, Time evolution of liquid drop impact onto solid, dry surfaces, Experiments in Fluids 33, (2002) 112-124.

[22] D. Quere, Wetting and Roughness, Annu. Rev. Mater. Res. 38, (2008) 71-99

[23] Bolleddula, D. A.,"Droplet impact and spreading of viscous dispersions and volatile solutions", Ph.D Dissertation, University of Washington, (2011).

[24] G.D. Martin, S.D. Hoath, I.M. Hutchings, Inkjet printing - the physics of manipulating liquid jets and drops, Journal of Physics: Conference Series 105, (2008), 012001.

[25] S. Doelle, R. Stannarius, Microdroplets Impinging on Freely Suspended Smectic Films: Three Impact Regimes, Langmuir 31(23), (2015) 6479-6486.

[26] H. Yoo, C. Kim, Experimental studies on formation, spreading and drying of inkjet drop of colloidal suspensions, Colloids and Surfaces A-Physicochemical and Engineering Aspects 468, (2015), 234-245.

[27] J. Philippi, P.Y. Lagree, A. Antkowiak, Drop impact on a solid surface: short-time self-similarity, Journal of Fluid Mechanics 795, (2016) 96-135.

[28] K. Kobayashi, K. Konno, H. Yaguchi, Early stage of nanodroplet impact on solid wall, Physics of Fluids 28(3), (2016) 032002.

[29] J.B. Lee, D. Derome, R. Guyer, Modeling the Maximum Spreading of Liquid Droplets Impacting Wetting and Nonwetting Surfaces, Langmuir 32(5), (2016) 1299-1308.

[30] W. Carter, G.C. Popell, J. Samuel, S. Mishra, Fundamental Study and Modeling of the Droplet Formation in Near-field Electrohydrodynamic Printing, ASME Journal of Micro and Nano Manufacturing, 2(2), (2014) 021005 (12 pages)

[31] N.S. Cheng, Formula for the Viscosity of a Glycerol-Water Mixture, Industrial and Engineering Chemistry Research, 47(9), (2008) 3285-3288.

[32] Physical Properties of Glycerine and Its Solutions, Glycerine Producers’ Association (1963). 
[33] C. Spackman, C. Frank, K. Picha, J. Samuel, 3D Printing of Fiber-reinforced Soft Composites: Process Study and Material Characterization, submitted to the Journal of Manufacturing Processes (Aug 18, 2015).

[34] S. Jung, I.M. Hutchings, The impact and spreading of a small liquid drop on a non-porous substrate over an extended time scale, Soft Matter 8, (2012) 2686-2696.

[35] S.R.L. Werner, J.R. Jones, A.H.J. Paterson, R.H. Archer, D.L. Pearce, Droplet impact and spreading: Droplet formulation effects, Chemical Engineering Science 62, (2007) 2336-2345.

[36] I. Ghai, J. Samuel, R.E. DeVor, S.G. Kapoor, Analysis of droplet spreading on a rotating surface and the prediction of cooling and lubrication performance of an atomization-based cutting fluid system, Journal of Manufacturing Science and Engineering 135(3), (2013), 031003 (12 Pages).

[37] L. Chen, E. Bonaccurso, Effects of surface wettability and liquid viscosity on the dynamic wetting of individual drops, Phys. Rev. E, Stat. Nonlinear Soft Matter Phys. 90, (2014) 022401, (9 pages).

[38] I.V. Roisman, R. Rioboo, C. Tropea, Normal impact of a liquid drop on a dry surface: model for spreading and receding, Proceedings of the Royal Society of London, Series A, (2002) 1411-1430.

[39] AccuDyne Testing, 〈https://www.accudynetest.com/polytable_03.html?sortby=contact_angle>

[40] Stratasys Objet TangoPlus FLX930 Safety Data Sheet, Section 9, page 5. 